\section{Prevention and treatment of Clostridium difficile infection}

\author{
Gianenrico Rizzatti, Gianluca Ianiro, \\ Giovanni Cammarota, \\ Antonio Gasbarrini
}

Department of Internal Medicine, Gastroenterology Division, A. Gemelli Hospital, Roma, Italy

\begin{abstract}
Clostridium difficile infection (CDI) is one of the most common intestinal infection often requiring hospitalization. Several risk factors are associated with the development of CDI with antibiotic treatments being the most common. Diagnosis is based on clinical suspicion, (typical symptoms associated with risk factors) and confirmatory tests, both of which are often inadequate leading to underestimation of the disease.

The management of CDI is based on fluid and nutritional support, avoidance of predisposing conditions and therapeutic interventions.

Therapy consists mainly in antibiotics with vancomycin and metronidazole being the most used, the former is usually reserved for severe cases or when metronidazole cannot be used. Evidence is increasing about the use of fidaxomicin, which is as effective as vancomycin but associated with lower rates of recurrences.

Lastly, fecal microbiota transplantation is now recommended by most guidelines for recurrent CDI obtaining extremely higher efficacy in comparison to standard treatments.
\end{abstract}

\section{Diagnosis and prevention}

Clostridium difficile infection (CDI) is one of the most prevalent intestinal infection in western countries with a reported pointprevalence of 17.2-19.0 per 10,000 patient bed-days in Europe. ${ }^{1}$ Furthermore, it has to be noted that occurrence of CDI is often underestimate due to inadequacy of standardizes testing and lack of clinical suspicion. ${ }^{1}$

Occurrence and spreading of CDI can be significantly limited by introduction of prevention measures. The most important elements are early recognition of CDI in patients with known risk factors, use of appropriate diagnostic tests and communication to the staff members of positive cases. Once CDI is diagnosed isolation precautions, protective clothing and appropriate cleaning of environmental and medical equipment are recommended. Alternatively, disposable single-use equipment can be used. Affected patients should be isolated in a single room or together with other patients diagnosed with CDI. Commonly, contact precautions should be continued for $48 \mathrm{~h}$ from symptoms resolution. Antibiotic stewardship and infection control programs are recommended as they are proven to be effective in reducing CDI incidence. However, routine screening for CDI in asymptomatic patients is discouraged as treatment of CD carriers is not recommended and antibiotic therapy is not effective in eliminating carriage.

\section{Treatments}

The principles of CDI management are discussed in the latest European ${ }^{2}$ and American guidelines. ${ }^{3}$ Management of CDI relies on three points: fluid and nutritional support, avoidance of predisposing conditions and therapeutic interventions.

Predisposing conditions include use of non-CDI specific antibiotics and protonpump inhibitors, which should be discontinued once CDI is diagnosed. All antibiotics can be associated with CDI, even CD-specific antibiotics. However, the most common antibiotics associated with development of CDI are clindamycin, cephalosporins and fluoroquinolones. Independently from the used agent, combination of two or more antibiotics as well as prolonged therapies also increase the risk of CDI.

Therapeutic interventions mainly involve the use of antibiotics. Recently, however, modulation of gut microbiota, especially by means of fecal microbiota transplantation (FMT), has proven to be a valuable therapeutic option, in particular, for recurrent CDI.

Severity assessment of CDI is of paramount importance as the treatment choice is based also on this parameter. Severe CDI is defined variably by the presence of one or more of the following: fever, hemodynamic instability and shock, respiratory failure, peritonitis, ileus, marked leukocytosis, renal function impairment, elevated serum lactate, reduced serum albumin.

In case of the first episode of non-severe CDI the recommended first line treatment consist of oral metronidazole. Other accepted regimens are oral vancomycin and fidaxomicin. Oral vancomycin is usually reserved for severe cases as it appears to be more effective. Oral vancomycin is also preferred in non-severe CDI in case of intolerance or allergy to metronidazole or in case of pregnancy or breastfeeding.

Furthermore, there is concern of
Correspondence: Gianenrico Rizzatti, Department of Internal Medicine, Gastroenterology Division, Catholic University of Rome, A. Gemelli Hospital, Largo Gemelli 8, 00168 Roma, Italy.

Tel./Fax: +39.06.30156018.

E-mail: gianenrico.rizzatti@gmail.com

Key words: Clostridium difficile; therapy; fecal microbiota transplant.

Contributions: the authors contributed equally.

Conflict of interest: the authors declare no potential conflict of interest.

Conference presentation: paper presented at $31^{\circ}$ Congresso Nazionale SIGOT - Genova, June 8-9, 2017.

Received for publication: 31 October 2017. Accepted for publication: 9 November 2017.

This work is licensed under a Creative Commons Attribution-NonCommercial 4.0 International License (CC BY-NC 4.0).

(C) Copyright G. Rizzatti et al., 2017

Licensee PAGEPress, Italy

Geriatric Care 2017; 3:7170

doi:10.4081/gc.2017.7170

increasing growth of vancomycin resistant enterococci with vancomycin use, whereas administration of metronidazole and, especially, fidaxomicin seems to be correlated with a lower risk. Finally, it has to be kept in mind that a course of metronidazole is considerably cheaper than a course of vancomycin. Notably, in case of non-severe CDI clearly associated with antibiotic therapy a wait and see strategy, consisting in discontinuation of the inducing antibiotic and close monitoring for $48 \mathrm{~h}$, might be pursued. Conversely, American guidelines advocate initiating empirical therapy for CDI regardless of laboratory test result.

Severe CDI is best managed with the administration of oral vancomycin. The recommended dosage of oral vancomycin is $125 \mathrm{mg}$ four times a day for 10 days. An alternative is represented by fidaxomicin, which proved to be not inferior in comparison to vancomycin however, data is still scant about its use in case of life-threating disease. Conversely, in the context of severe disease, metronidazole administration is discouraged as the cure rates appear to be lower in comparison to vancomycin.

Data about the use of fidaxomicin in CDI is increasing with promising results. In fact, a recent meta-analysis evaluated the efficacy of fidaxomicin in comparison to vancomycin and metronidazole in CDI. ${ }^{4}$ Two phase 3 RCTs comparing fidaxomicin 
and vancomycin were included. For comparison between fidaxomicin and metronidazole indirect data were obtained from 3 studies confronting metronidazole and vancomycin as direct evidence is not available yet. Results indicate similar cure rates for fidaxomicin in comparison with vancomycin even after stratification for disease severity. However, lower recurrence rates were observed for fidaxomicin in comparison with vancomycin.

Similar conclusions were drawn for comparison between fidaxomicin and metronidazole from the indirect data available.

Based on these results cost-efficacy studies were carried and results indicate that fidaxomicin is cost-effective in patients with severe CDI or with a first recurrence of CDI. ${ }^{5}$

When oral administration of antibiotics is not feasible intravenous administration of metronidazole is the preferred choice, possibly in conjunction with intracolonic or nasogastric administration of vancomycin.

American authors prefer to distinguish between a severe disease, defined by a reduced serum albumin and leukocytosis or abdominal tenderness, from a severe and complicated stage of disease further characterized by signs of end organ failure, fever, admission to intensive care unit, mental status change, elevated serum lactate or marked leukocytosis.

In this regard, American guidelines indicate in case of severe complicated CDI the administration of oral vancomycin associated with intravenous metronidazole as the treatment of choice. The further addition of rectal vancomycin is advocated in case of complicated CDI with ileus and toxic megacolon.

\section{Use of probiotics}

Data about the use of probiotics is still limited. At the moment, while some studies obtained positive results, both European and American guidelines for CDI management conclude that there is insufficient data to support the administration of probiotics. Conversely WGO guidelines for probiotics and prebiotics do give indications for the administration of probiotics for the prevention of CDI-associated diarrhea. ${ }^{6}$

In this context, a possible emerging option is represented by the administration of spores of nontoxigenic CD strain M3 (NTCD-M3). In fact, a RCT was recently conducted evaluating the safety and efficacy of the administration of this strain in the prevention of CDI recurrence. ${ }^{7}$ Authors found that administration of NTCD-M3 was safe as both mild and serious treatment related adverse effects were similar between the intervention group and the placebo group. Furthermore, recurrence of CDI after recovery from an antibiotic course was significantly lower in the arm that received NTCDM3 in comparison to placebo, $11 \%$ and 30\% recurrence rates, respectively. Importantly, recurrence rates where even lower in patients that, after NTCD-M3 administration showed a fecal colonization by this strain in comparison to those that did not achieved fecal colonization.

Futhermore, it has been demonstrated that specific microbiota component are associated with resistance to CD colonization, in particular Clostridium scindens (CS) ${ }^{8}$ The mechanisms behind this phenomenon probably involve bile acids metabolism. In fact, it has been demonstrated that $\mathrm{CS}$ is able to transform primary bile acids into secondary bile acids thanks to a specific enzyme ( $7 \alpha-$ hydroxysteroid dehydrogenase). Secondary bile acids are then able to inhibit $\mathrm{CD}$ growth. Possibly, this data might contribute in the near future, to the development of new therapeutic agents for CDI.

\section{Recurrent Clostridium difficile infection}

The term recurrent CDI (RCDI) refers to the occurrence of CDI no later than 8 weeks after the onset of a previous, resolved, episode. First recurrence can be managed, with the same efficacy, with administration of vancomycin, metronidazole or fidaxomicin. However, treatment of the first recurrence with fidaxomicin is associated with fewer subsequent recurrences than with vancomycin. Furthermore, in case of severe CDI vancomycin should be preferred. From the second recurrence a pulsed vancomycin regimen should be used. The same therapeutic strategies are used in case of CDI presenting with factors associated with an increased risk of a recurrent behavior. These factors are age above 65 years, continuation of non-CDI specific antibiotics, more than one recurrence, continuation of proton pump inhibitors and disease severity at the onset.

After the first recurrence, subsequent non-severe recurrences can be managed with oral vancomycin or fidaxomicin.

\section{Fecal microbiota transplantation}

As already mentioned, another nonantibiotics strategy used in the management of multiple recurrent CDI include the use of
FMT. Data on the efficacy of FMT in RCDI is rapidly increasing. A recent systematic review $^{9}$ evaluated the efficacy of FMT in RCDI. The studies evaluated were mostly case-series with only 2 randomized controlled trials (RCTs). One RCT compared FMT with vancomycin or vancomycin associated with bowel lavage. Percentages of symptom resolution were significantly higher in the FMT group compared with the other two interventions. In case-series studies patients characteristics and reported outcomes were more heterogeneous. Notably, symptoms resolution rates might have been biased by the commonly reported administration of antibiotics before FMT. However, overall symptom resolution without recurrence was seen in $85 \%$ of RCDI cases treated with FMT.

Similar results were obtained in another RCT. ${ }^{10}$ In this trial patients with RCDI were randomized to receive either FMT or standard vancomycin regimen. The protocol provided that fecal infusions of microbiota were repeated every 3 days until the resolution of colitis. The primary end point was symptom resolution at 10 weeks from the end of the assigned treatment. In the FMT group $65 \%$ of patients reached the primary end point with a single FMT infusion, while with multiple infusions the percentage reaches $90 \%$.

In comparison, patients in the vancomycin arm met the primary end point only in the $26 \%$ of cases.

European guidelines recommend FMT in combination with antibiotic treatment after the first relapse while American guidelines recommend that FMT should be considered in case of a third recurrence of CDI after a pulsed vancomycin regimen.

Due to the great efficacy of FMT in CDI and its potential use in other disorders a European consensus conference on the use of FMT in clinical practice was recently published in order to give evidence-based recommendations about indications, methodology for the use of this procedure. ${ }^{11}$ In accordance, FMT is recommended as a treatment option for both mild and severe RCDI with high quality of grading and strong recommendation as evidence is available from several RCTs. Furthermore, FMT can be considered also in refractory CDI, however, in this case the quality of evidence is low and further study are awaited to give more robust results on this matter. Conversely, FMT cannot be recommended, at the moment, for the treatment of the first episode of CDI.

\section{The role of surgery}

Finally, surgery and, in particular, total colectomy, is still required in selected cases 
or where medical therapies fail. Indications to perform surgery are perforation, worsening clinical conditions despite appropriate antibiotic therapy, toxic megacolon, acute abdomen and ileus. Several risk factors have been identified and demonstrated to be associated with higher rates of mortality including shock, increased lactate, organ failure and need for intubation and ventilation. If these factors are present earlier referral to surgery might be warranted.

\section{References}

1. Davies KA, Longshaw CM, Davis GL, et al. Underdiagnosis of Clostridium difficile across Europe: the European, multicentre, prospective, biannual, point-prevalence study of Clostridium difficile infection in hospitalised patients with diarrhoea (EUCLID). Lancet Infect Dis 2014;14:1208-19.

2. Debast SB, Bauer MP, Kuijper EJ. European Society of Clinical Microbiology and Infectious Diseases: update of the treatment guidance document for Clostridium difficile infection. Clin Microbiol Infect 2014;20 Suppl 2:1-26.

3. Surawicz CM, Brandt LJ, Binion DG, et al. Guidelines for diagnosis, treatment, and prevention of Clostridium difficile infections. Am J Gastroenterol 2013; 108:478-98; quiz 99.

4. Cornely OA, Nathwani D, Ivanescu C, et al. Clinical efficacy of fidaxomicin compared with vancomycin and metronidazole in Clostridium difficile infections: a meta-analysis and indirect treatment comparison. J Antimicrob Chemother 2014;69:2892-900.

5. Nathwani D, Cornely OA, Van Engen AK, et al. Cost-effectiveness analysis of fidaxomicin versus vancomycin in Clostridium difficile infection. J Antimicrob Chemother 2014;69:2901-12.

6. Guarner F, Khan AG, Garisch J, et al. World Gastroenterology Organisation Global Guidelines: probiotics and prebiotics October 2011. J Clin Gastroenterol 2012;46:468-81.

7. Gerding DN, Meyer T, Lee C, et al.
Administration of spores of nontoxigenic Clostridium difficile strain M3 for prevention of recurrent $C$. difficile infection: a randomized clinical trial. JAMA 2015;313:1719-27.

8. Vogt SL, Pena-Diaz J, Finlay BB. Chemical communication in the gut: effects of microbiota-generated metabolites on gastrointestinal bacterial pathogens. Anaerobe 2015;34:106-15.

9. Drekonja D, Reich J, Gezahegn S, et al. Fecal microbiota transplantation for Clostridium difficile Infection: a systematic review. Ann Intern Med 2015; 162:630-8.

10. Cammarota G, Masucci L, Ianiro G, et al. Randomised clinical trial: faecal microbiota transplantation by colonoscopy vs. vancomycin for the treatment of recurrent Clostridium difficile infection. Aliment Pharmacol Ther 2015;41:835-43.

11. Cammarota $G$, Ianiro $G$, Tilg $H$, et al. European consensus conference on faecal microbiota transplantation in clinical practice. Gut 2017;66:569-80. 